\title{
Supramolecular Heterocatemers and Their Role in Co-crystal Design
}

Joanna A. Bis, Olga L. McLaughlin, Peddy Vishweshwar, Michael J. Zaworotko*

Department of Chemistry, University of South Florida, CHE205, 4202 East Fowler Avenue, Tampa, Florida, 33620

\section{Supporting Information}

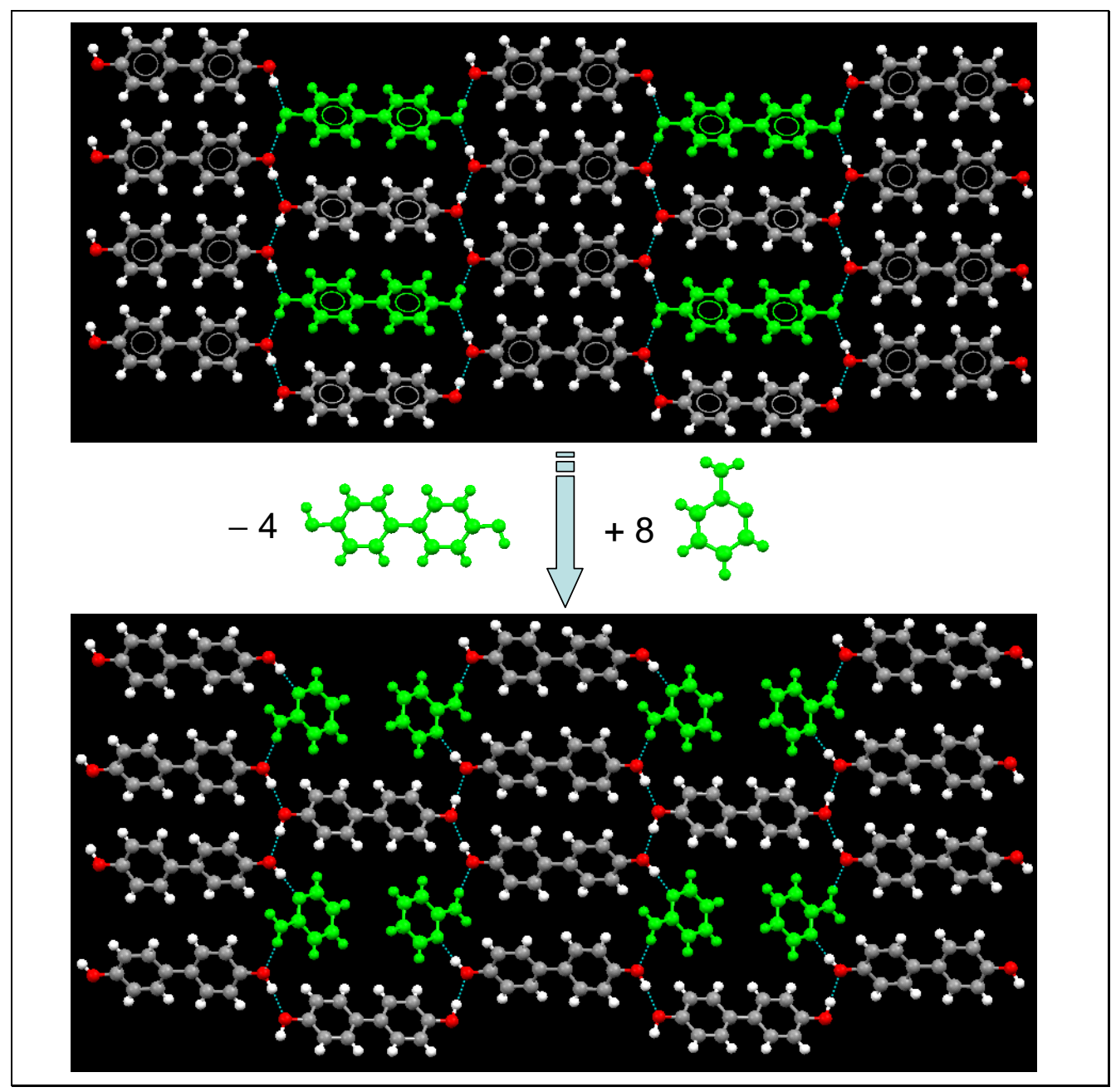

Figure S1. From "4,4'-biphenol crystal" to "(4,4'-biphenol) $)_{3} \bullet(2 \text {-aminopyridine })_{2}, \mathbf{1}$ cocrystal" through supramolecular heterocatemers. Notice that arrangement of molecules in pure 4,4'-biphenol and co-crystal $\mathbf{1}$ is similar. 


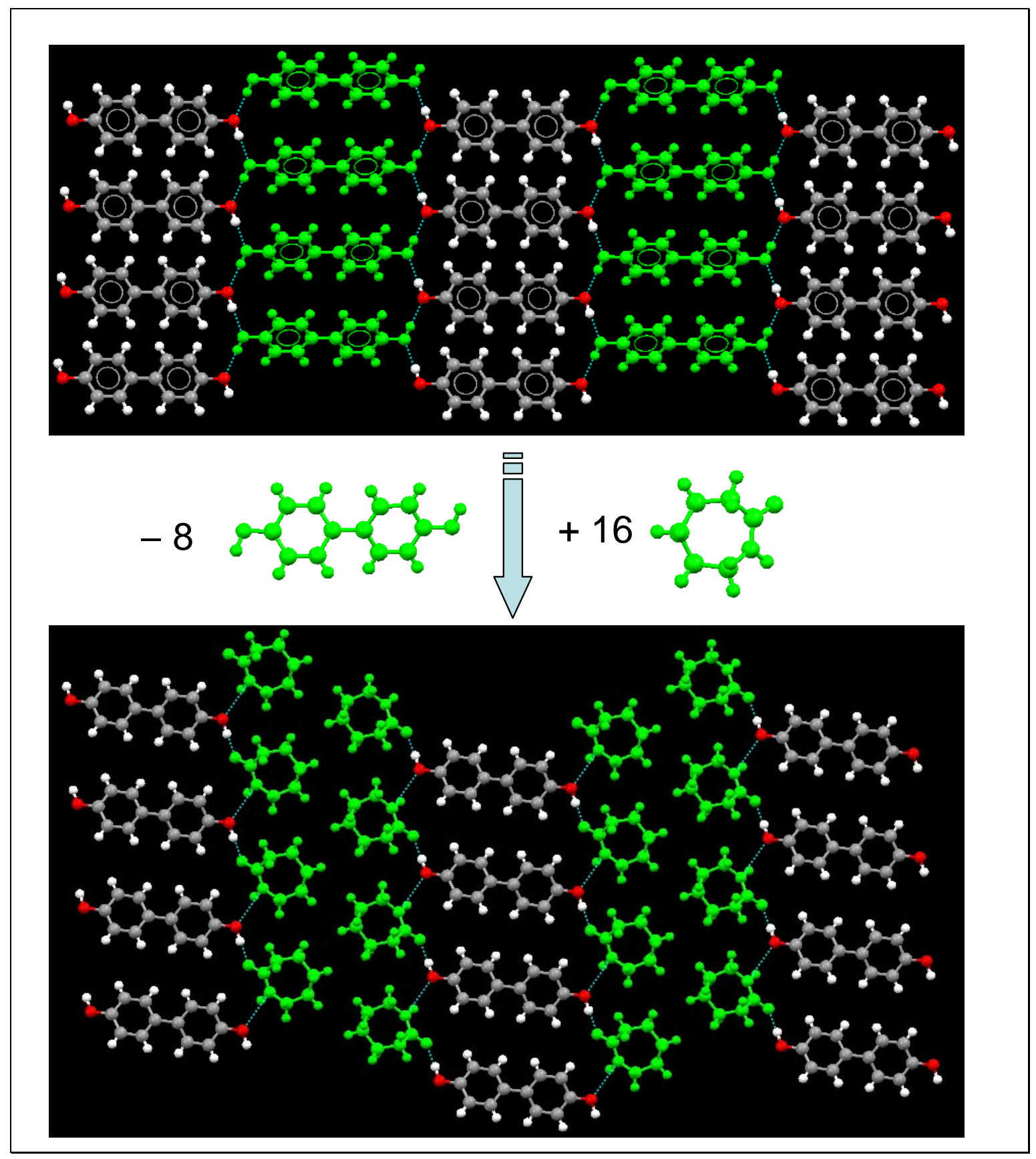

Figure S2. From "4,4'-biphenol crystal” to "(4,4'-biphenol)•(Caprolactam $)_{2}, 2$ cocrystal" through supramolecular heterocatemers. Notice that arrangement of molecules in pure 4,4'-biphenol and co-crystal $\mathbf{2}$ is similar. 


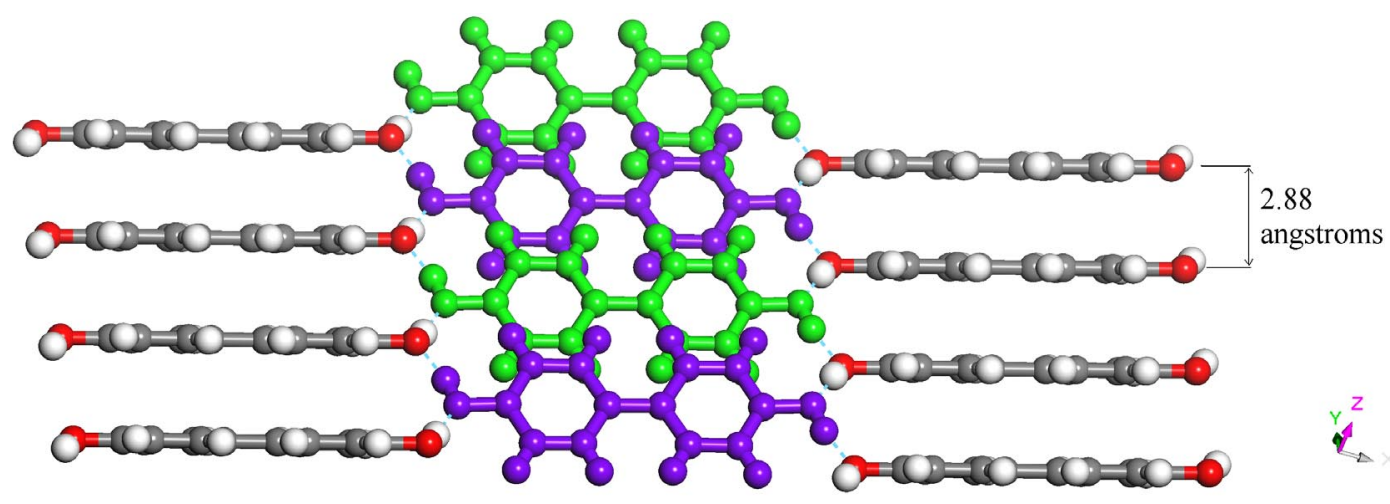

Figure S3. Figure 1 view down $b$-axis shows that adjacent 4,4 '-biphenols along supramolecular homocatemer are separated by $2.88 \AA$.
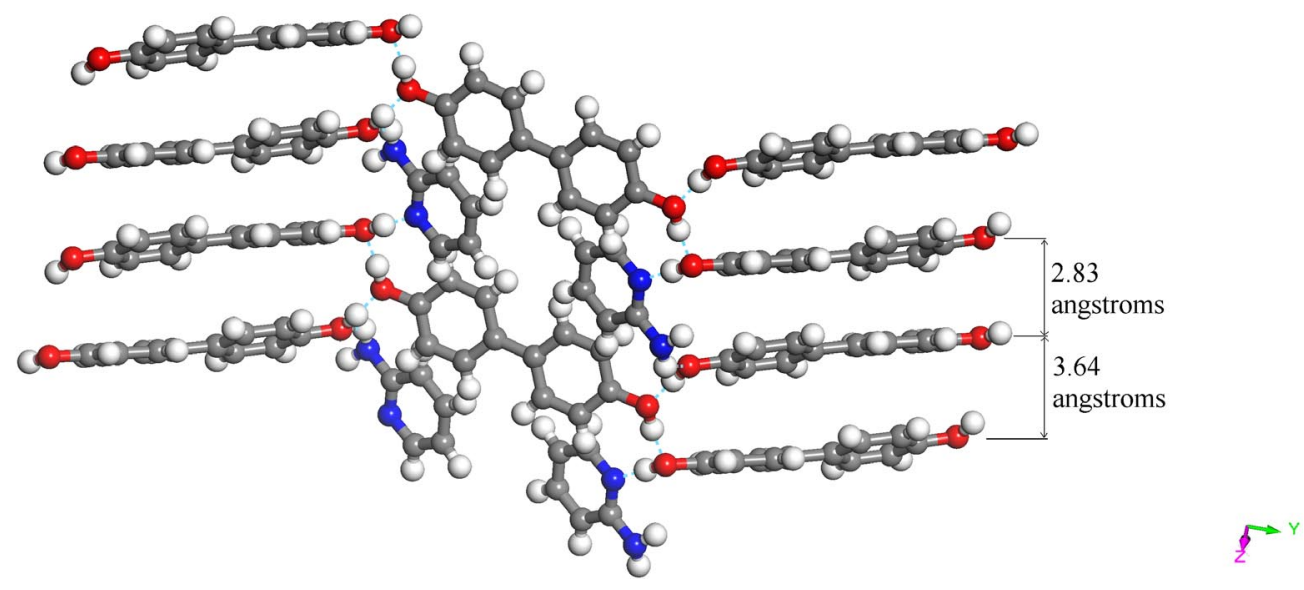

(a)

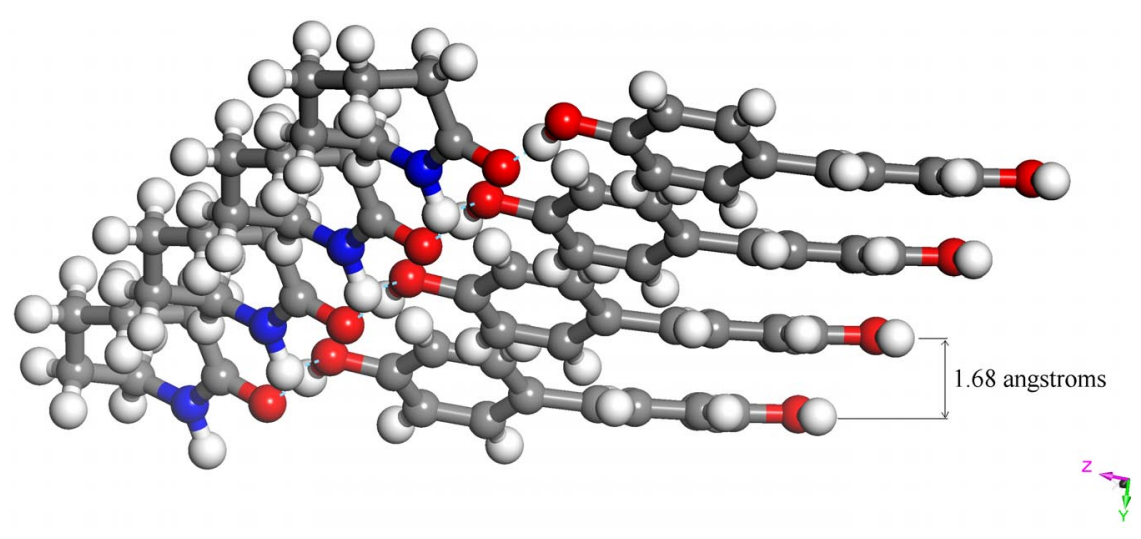

(b)

Figure S4. (a) Crystal packing of co-crystal 1 along $a$-axis shows that 4,4'-biphenols are separated by $2.83 \AA$ (with a 2-aminopyridine) and $3.64 \AA$ (with a 4,4'-biphenol) in a supramolecular heterocatemer. (b) Crystal packing of co-crystal 2 shows that adjacent 4,4'-biphenols along supramolecular heterocatemer are separated by $1.68 \AA$. 
Table 1. Hydrogen bonds for co-crystal 1 [Å and ${ }^{\circ}$ ].

\begin{tabular}{lcccc}
\hline D-H...A & d(D-H) & d(H...A & d(D...A & $<($ DHA $)$ \\
\hline $\mathrm{O}(31)-\mathrm{H}(31) \ldots \mathrm{O}(2) \# 2$ & 0.96 & 1.84 & $2.788(2)$ & 168.8 \\
$\mathrm{~N}(22)-\mathrm{H}(22 \mathrm{~A}) \ldots \mathrm{O}(1) \# 3$ & 0.91 & 2.45 & $3.134(3)$ & 131.8 \\
$\mathrm{O}(2)-\mathrm{H}(2) \ldots \mathrm{N}(21) \# 4$ & 0.92 & 1.78 & $2.697(2)$ & 171.9 \\
$\mathrm{O}(1)-\mathrm{H}(1) \ldots \mathrm{O}(31) \# 5$ & 0.97 & 1.84 & $2.811(2)$ & 173.8 \\
& & & & \\
\hline
\end{tabular}

Symmetry transformations used to generate equivalent atoms:

$\# 1-\mathrm{x}+1,-\mathrm{y},-\mathrm{z}+2 \quad \# 2-\mathrm{x}+1,-\mathrm{y}+1,-\mathrm{z}+1 \quad \# 3 \mathrm{x}, \mathrm{y}, \mathrm{z}+1$

$\# 4-\mathrm{x}+2,-\mathrm{y}+1,-\mathrm{z}+1 \quad \# 5 \mathrm{x}, \mathrm{y}, \mathrm{z}-1$

Table 2. Hydrogen bonds for co-crystal 2 [ and $\left.^{\circ}\right]$.

\begin{tabular}{lcccc}
\hline D-H...A & $\mathrm{d}(\mathrm{D}-\mathrm{H})$ & $\mathrm{d}(\mathrm{H} \ldots \mathrm{A})$ & $\mathrm{d}(\mathrm{D} \ldots \mathrm{A})$ & $<(\mathrm{DHA})$ \\
\hline $\mathrm{O}(1)-\mathrm{H}(1) \ldots \mathrm{O}(11) \# 2$ & 0.99 & 1.64 & $2.6285(14)$ & 172.6 \\
$\mathrm{~N}(11)-\mathrm{H}(11) \ldots \mathrm{O}(11) \# 3$ & 1.07 & 1.98 & $3.0142(15)$ & 161.8 \\
& & & & \\
\hline
\end{tabular}

Symmetry transformations used to generate equivalent atoms:

$\# 1-x, y,-z+1 / 2 \quad \# 2 x+1 / 2,-y+1 / 2, z+1 / 2 \quad \# 3-x+1 / 2,-y+1 / 2,-z$

Table 3. Hydrogen bonds for co-crystal 3 [ and $\left.^{\circ}\right]$.

\begin{tabular}{lcccc}
\hline $\mathrm{D}-\mathrm{H} \ldots \mathrm{A}$ & $\mathrm{d}(\mathrm{D}-\mathrm{H})$ & $\mathrm{d}(\mathrm{H} \ldots \mathrm{A})$ & $\mathrm{d}(\mathrm{D} \ldots \mathrm{A})$ & $<(\mathrm{DHA})$ \\
\hline $\mathrm{O}(1)-\mathrm{H}(1) \ldots \mathrm{O}(21) \# 1$ & 0.945 & 1.728 & 2.665 & 170.55 \\
$\mathrm{O}(2)-\mathrm{H}(2) \ldots \mathrm{O}(11) \# 2$ & 1.020 & 1.686 & 2.674 & 161.68 \\
$\mathrm{~N}(11)-\mathrm{H}(11) \ldots \mathrm{O}(11) \# 3$ & 0.982 & 1.968 & 2.950 & 176.86 \\
$\mathrm{~N}(21)-\mathrm{H}(21) \ldots \mathrm{O}(1)$ & 0.860 & 2.136 & 2.956 & 159.28 \\
& & & & \\
\hline
\end{tabular}

Symmetry transformations used to generate equivalent atoms:

$\# 1 \mathrm{x}-1, \mathrm{y}, \mathrm{z} \quad \# 2 \mathrm{x}, \mathrm{y}-1, \mathrm{z} \quad \# 3-\mathrm{x}+1,-\mathrm{y}+1,-\mathrm{z}+1$ 